\title{
El sistema de incentivo y estructura salarial en la profesionalización docente. El caso del Sistema Nacional de Evaluación del Desempeño Docente
}

\author{
The Incentives and Salary Structure System in Teacher \\ Professional Development. The Case of the National Teacher \\ Performance Evaluation System
}

Christian Loyola Bustos*

\section{RESUMEN}

En el presente trabajo se analizará, a partir de la revisión de la liPalabras clave: teratura especializada, la experiencia de la Ley 19.410, que crea y norma el funcionamiento del Sistema Nacional de Evaluación de Desempeño Docente en relación con los sistemas de incentivos y estructura salarial del profesorado. Lo que se pretende con esta nota es alcanzar una panorámica que nos permita comprender y entender la importancia de esta Ley, el momento histórico en el que surge, su fase de desarrollo en el marco de las políticas educativas, regulaciones, impacto y narrativas que se desencadenan en esta política educativa.
ABSTRACT
This article will apply a specialized literature review to analy- Key words: ze the experience of Law 19,410, which creates and regulates educational policy, the operation of the National Teacher Performance Evaluation System, in relation to teacher incentives and salary structure systems. The objective is to obtain an overview that will allows us to understand and comprehend the importance of this Law, the moment in time when it was passed, its development pha- National Teacher Performance Evaluation System (SNED), teacher professional incentives, teacher professional política educativa, Sistema Nacional de Evaluación Docente (SNED), incentivo profesional docente, profesionalización docente.

\footnotetext{
* Profesor de Historia, licenciado en Historia. Magíster en Política Educativa y en Liderazgo y Gestión de las Organizaciones Escolares. Pontificia Universidad Católica de Valparaíso. Contacto: christian.loyola@pucv.cl ORCID: https://orcid.org/0000-0003-4792-9936
} 
El sistema de incentivo y estructura salarial en la profesionalización docente | Loyola

se within the framework of educational policy, regulations, im- development. pact and narratives unleashed within this educational policy. 


\section{Introducción}

El presente trabajo tiene por objetivo principal conocer y analizar la experiencia del Sistema Nacional de Evaluación Docente (SNED) (Ley 19.410) y su importancia en la política docente en Chile. El estudio no está centrado en un análisis exhaustivo y crítico de la Ley, sino más bien en el impacto, percepciones y narrativas a partir de la implementación de la ley. Este estudio se fundamenta en una problemática de discusión actual y permanente, que tiene relación con la profesionalización de la carrera docente y el propósito de aseguramiento de la calidad. Claramente, la política docente es uno de los aspectos más relevantes de la política educativa chilena. Si bien los avances producidos durante los últimos años han permitido estructurar de mejor manera una política docente, sigue habiendo hasta hoy algunas limitaciones.

De la misma manera, lo que se pretende en este trabajo es revisar, desde una perspectiva panorámica, la Ley 19.410 y su impacto en la estructura salarial de los docentes a partir de los incentivos. Por ello, la metodología de esta observación se sustenta en una revisión bibliográfica (o de literatura) que, a partir del análisis de fuentes secundarias vinculadas principalmente a la Formación Inicial Docente, regulación e incentivos de los profesores, y políticas educativas, entre otras, forman la base teórica de este análisis.

La pregunta eje que guía este trabajo es: ¿cuál es la importancia e impacto de la ley que crea el SNED en la política educativa docente? Para responder nos hemos propuesto analizar los siguientes objetivos específicos:

a) conocer la estructura salarial de los profesores durante las décadas del 80 y del 90;

b) identificar el SNED como un sistema que impacta en las remuneraciones de los docentes sus regulaciones y narrativas;

c) analizar si los incentivos tienen una relevancia en la calidad de la educación,

d) y determinar, a partir de la visión de distintos investigadores, los posibles cambios y regulaciones en los sistemas salariales e incentivos de los docentes. 


\section{La política docente. Contexto y estructura salarial}

A continuación, analizaremos los principales cambios, modificaciones y transformaciones que han tenido las políticas de profesionalización docente en la estructura salarial a partir de las décadas del 80 y del 90 .

\subsection{La reforma promercado y la profesionalización docente.}

El devenir de la globalización ha impactado fuertemente en las políticas educativas, especialmente en países en vías de desarrollo, como es el caso chileno. Este impacto ha sido reflejado en la incorporación de nuevas formas de orientaciones basadas en el mercado, la competitividad, el consumo y la privatización, entre otras. Al respecto, Carnoy (1999) señala que la globalización impacta a la educación en el financiamiento, en el mercado laboral y en la calidad. Desde esta perspectiva, emerge con fuerza la idea de privatización de los sistemas educativos. De acuerdo con Ball y Youdell (2008), esta privatización se hace de manera encubierta. En el caso chileno, estas medidas fueron impulsadas por el golpe militar de 1973 (Carnoy \& González, 2004; Cox, 2005; Bellei, 2015; Corvalán \& García Huidobro, s.f.; Ávalos, 2002; entre otros). La junta militar, con fundamentos provenientes desde la economía liberal, inspirados en expertos que suponen un progreso en la educación (Beech, 2006), producen un cambio radical en las formas de regulación del sistema educativo, lo que traerá por consecuencia un proceso de complejización al sistema, generado por los distintos procesos selectivos (Corvalán \& García Huidobro s.f.). De acuerdo con Cox (2005), los propósitos gubernamentales en los años 80 estuvieron dirigidos al logro de una mayor eficiencia en el uso de los recursos, a través de la competencia entre establecimientos por matrícula; el traspaso de funciones desde el Ministerio de Educación a los poderes locales representados en el Municipio; la disminución del poder de negociación del gremio docente; una mayor participación del sector privado en la provisión de la educación, y una mayor cercanía con la educación media técnico-profesional. Bellei y Vanni (2015) afirman que "cada una de estas medidas sería por sí misma una política enormemente novedosa para cualquier sistema escolar tradicional; todas juntas fueron una revolución para el sistema chileno" (p. 24). No obstante, ¿cuál es la relación entre el Estado y el gremio? ¿Cuáles fueron sus efectos? 
De acuerdo con el Programa Interdisciplinario de Investigaciones en Educación (PIIE) (1984), durante los años 1973 y 1974 el Estado "desarrolló una política de control y depuración del magisterio, inspirada en motivaciones de seguridad nacional" (p. 156), pero también, paralelamente, se van a concretar dos realizaciones principales: la fundación del Colegio de Profesores y la estructuración de la carrera docente. De acuerdo con los autores del PIIE (1984), "Los sueldos y salarios, en general, fueron afectados gravemente por el fuerte incremento de la inflación en los últimos meses de 1973. El Gobierno Militar reajustó las remuneraciones en proporción al alza del costo de la vida" (p. 158). A partir de la movilización gremial por la reivindicación de ciertos derechos, surgirá con fuerza la expresión de una "política dignificadora”. En 1979 se publica la Ley 2.327, que crea la carrera docente y regula su ejercicio (Núñez, 2002). Un elemento de la Ley es que "ya se establecía estimular la superación y la eficiencia mediante un sistema de remuneraciones acorde con sus méritos y antigüedad" (PIIE, 1984, p. 165). De esta forma, las remuneraciones quedaron compuestas por el sueldo asignado al grado en la Escala Única de Remuneraciones, una asignación muy pequeña por antigüedad, cada dos años según el Decreto Ley 249 de 1973, y una asignación docente para quienes tenían el título profesional (PIIE, 1984). Finalmente, la Ley de Carrera Docente no fue percibida como un aporte, sino más bien como un proceso de jerarquización y disciplinamiento de los profesores. En 1980 la Ley de Carrera Docente sufrirá una serie de modificaciones, dando paso a una fuerte resistencia por parte de los docentes (Hargreaves \& Shirley, 2012).

El proceso de traspaso de la educación estatal a las municipalidades significó abandonar el régimen de funcionario público para pasar a la condición de trabajadores del sector privado con que se les contrató en los establecimientos municipalizados (PIIE, 1983). Estas transformaciones tienen una serie de implicancias, algunas de ellas son los cambios en la visión de algunos agentes educativos (Ball \& Youdell, 2008).

De acuerdo con Mizala y Romaguero (2002), en ese momento los profesores perdieron su condición de trabajadores públicos y comenzaron a regirse por el Código del Trabajo, válido para los empleados del sector privado, sin cláusulas especiales, ni siquiera respecto de remuneraciones. Núñez (2002), al respecto, afirma que el traspaso a los municipios y la pérdida de su condición como trabajadores públicos fue un cambio 
extremadamente duro para los docentes, quienes nunca lo aceptaron, ya que iba en contra de la cultura docente. Este cambio o traspaso también tuvo su espejo en las remuneraciones, en las que se perdió todo tratamiento específico en virtud la labor docente. Con el Decreto Ley 2.200 se regularon las nuevas condiciones entre "empleadores y trabajadores": los educadores transferidos desde el sector público al sector privado (municipalidades) perdieron todos los beneficios contenidos en la Ley de Carrera Docente. No olvidemos tampoco a los profesores particulares: éstos también se vieron fuertemente perjudicados con la Ley 18.018. Durante la década del 80 el sueldo de los profesores cayó un tercio en términos reales (Cox, 2005), generando un legado lleno de desigualdades y haciendo efecto en la masificación de la escolarización, los niveles educativos, la calidad en la educación, el rol de las políticas públicas, entre otros (Tenti, 2011).

\subsection{La tercera vía en la educación. Las décadas de los 90 y las mejoras.}

Para algunos investigadores, como Iván Núñez (2002) y Beatrice Ávalos (2002), la década de los 90 forma parte de una "segunda profesionalización", permitiendo una serie de reformas estructurales, principalmente en los ámbitos de calidad y equidad, evaluación de logros y aprendizajes, y el desarrollo profesional docente (Gajardo, 2012). El Estado tomará un rol más preponderante en las decisiones educativas, estableciendo una serie de programas de mejoramiento ${ }^{2}$, pero ¿qué ocurre con los profesores? ¿Cambia su estructura salaria? ¿Cuál es su impacto?

Sin duda que durante los 90 se establecieron condiciones favorables para el ejercicio docente, reflejado, entre otros aspectos, en los ámbitos salarial y laboral. Las remuneraciones incrementaron desde un 145 a $170 \%$ real. Esta alza salarial tiene su marco metodológico en el Estatuto Docente (Ley 19.070) de 1991, que va a establecer una regulación nacional de las condiciones de empleo, tales como jornada de trabajo, horarios máximos, régimen de vacaciones, remuneraciones y otros (Cox, 2005). De acuerdo con Mizala y Romaguera (2002), "el Estatuto Docente, aprobado en 1991, volvió a centralizar decisiones sobre contratos y remuneraciones de los profesores de establecimientos municipales" (p. 3).

2 Por ejemplo, P-900; MECE-Básica; MECE-Rural; MECE-Media (Bellei y Vanni, 2015). 
Durante 1995 el Estatuto aprobó una serie de nuevas disposiciones que flexibilizaron la movilidad de la planta docente. La reforma al Estatuto Docente (Ley 19.410) de 1995 estableció un nuevo aumento de las remuneraciones de los profesores por vía de bonificación adicionales (Ávalos, 2005). El Estatuto Docente se aplica a todos los profesionales de la educación. Sin embargo, configura de manera diferenciada los contratos de los docentes dependiendo de donde trabajan. Aquellos que se desempeñan en establecimientos municipales se rigen por el tipo de contrato que se denomina "carrera docente". En contraste, en el mismo Estatuto se establece que aquellos que se desempeñan en el sector particular se rigen por un contrato privado, regulado por las normas del Código del Trabajo que se aplica a todos los trabajadores del sector privado, excepto en determinadas normas referidas a la jornada laboral, los feriados legales y la terminación del contrato (Mizala \& Romaguera, 2002, 2000a, 2000b, 2005; González, 1999).

Un elemento interesante son el conjunto de incentivos monetarios, o bonos salariales, a los cuales los profesores pueden acceder. Dentro de los incentivos más importantes, por medio del Sistema Nacional de Evaluación de Desempeño (SNED), destacamos el de la "antigüedad", la realización de cursos de perfeccionamiento o trasladarse a zonas geográficas de difícil desempeño.

El SNED hay que comprenderlo de acuerdo con la fase de desarrollo en la cual se enmarca, con su narrativa de por medio. Esa fase corresponde a una regulación del mercado, conocida también como la "tercera vía" en educación, en la que se ejecutan, por parte de los gobiernos centrales, diversos planes de mejoras. No se está hablando de "reformas" sino más bien de "mejoras": dadas las condiciones, es era lo que se podía hacer y se trató de implementar de las mejores maneras. Para Bellei (2015), durante los años 1996-2005 se producirán reformas de la calidad y equidad educacional. Justamente, uno de los ejes de esta "modernización" fue la inclusión de un heterogéneo paquete de medidas de apoyo a la profesión docente ${ }^{3}$, entre ellas estaban los

3 Entre ellas se creó un programa de fortalecimiento de la formación inicial docente, que entregó recursos a las universidades para mejorar su infraestructura y equipamiento, y renovar su curriculum y personal académico. Se implementó un programa masivo de perfeccionamiento docente, que incluyó cursos más o menos tradicionales en torno a la reforma curricular, y se inició un programa de pasantías para docentes en el extranjero (Bellei, 2016, p. 30.). 
incentivos monetarios hacia los profesores, los que incluyeron bonos mensuales, creándose el SNED.

\section{Ley 19.410. El Sistema Nacional de Evaluación de Desempeño (SNED) como incentivo y mejora salarial en la profesionalización docente}

Respecto del SNED, fueron de vital importancia las revisiones de los trabajos de Mizala y Romaguera (2000c; 2003 y 2005).

El SNED se empieza a implementar a partir de 1996 bajo la ley 19.410: "esta identifica a los colegios que reciben subvención estatal (tanto particulares subvencionados como municipales) de mejor desempeño en cada región del país" (2003, p. 4). El SNED introduce dos elementos adicionales en las reformas educativas: "(i) posibilita una mejor información sobre el resultado educativo de los alumnos; (ii) establece un incentivo a los docentes para mejorar el resultado del proceso educativo, lo que constituye un incentivo a la oferta" (2005, p. 543; 2000a, p. 26). No obstante, ¿qué estaba ocurriendo en la política educativa que hizo necesaria la implementación de la SNED? ¿Por qué va a surgir finalmente la ley? ¿Cuál es la narrativa educativa que existe detrás de la política? El desarrollo de esta política educativa se relaciona con que:

El sistema educacional chileno, luego de la reforma de los años 80, poseía un incentivo a la demanda: la elección de colegios por parte de alumnos y apoderados, asociados a la existencia de una subvención por matrícula destinada a establecimientos subvencionados. El SNED introduce un incentivo adicional, un incentivo a la oferta que, asociado directamente a los docentes, es un complemento importante del actual sistema educativo. Esto es así porque el incentivo a mejorar calidad vía la competencia entre colegios por captar alumnos no es suficiente; por una parte, dado que, en muchos lugares, por razones de tamaño de las ciudades, accesibilidad geográfica $\mathrm{u}$ otras no existen alternativas educacionales $\mathrm{y}$, por otra parte, debido a que la información de que disponen los padres para elegir colegios es insuficiente, lo que limita la racionalidad de sus decisiones al respecto. (Mizala \& Romaguero, 2000c, p. 13).

¿Cómo se obtienen los datos y comparaciones? El SNED es un sistema de evaluación de los docentes que tiene como un componente 
principal los resultados académicos de los alumnos, medidos a través de la prueba SIMCE. En palabras de los autores, lo que se espera es "construir grupos homogéneos", caracterizados por variables socioeconómicas a escala regional.

Debido a las diferencias en las condiciones que enfrentan los establecimientos educacionales, los rankings de establecimientos a través de los cuales se otorga la subvención por desempeño de excelencia (SNED) no se realizan directamente sobre la base de los puntajes brutos. Se busca comparar establecimientos de similares características, tanto socioeconómicas como geográficas. Para ello se construyen "grupos homogéneos" a nivel regional, caracterizados por variables socioeconómicas y geográficas similares y sólo se comparan establecimientos que pertenezcan a un mismo grupo. Los grupos homogéneos se calculan a nivel regional, el número de grupos varía de región en región, dependiendo de su tamaño y heterogeneidad. (Mizala \& Romaguera, 2005, p. 544).

En relación con los aspectos positivos del SNED, las autoras han rescatado, entre otras cosas, que:

El SNED busca, por una parte, mejorar la información disponible para los padres de familia y las autoridades educacionales y; por otra parte, cambiar la estructura de las remuneraciones docentes. Estas, en general, tienen poca varianza entre los profesores, lo que no refleja diferentes niveles de esfuerzo y habilidades, de hecho, los aumentos salariales responden principalmente a la antigüedad. (Mizala \& Romaguera, 2003, p. 17).

- Por su parte, el que un establecimiento educacional sea público (municipal) o privado con financiamiento público (particular subvencionado) no juega ningún rol en la obtención del premio SNED (Mizala \& Romaguera, 2005).

- El SNED es quien entrega los resultados más equitativos, al compararlo con indicadores alternativos que podrían ser utilizados para seleccionar establecimientos a ser premiados ${ }^{4}$ (Mizala \& Romaguera, 2003).

4 Un elemento a tener en cuenta es que, a partir de la medición y los datos entregados por el SNED, la desigualdad de elección de escuela se reduciría provocando así menos segregación. 


\section{1 ¿Cuál es el impacto del SNED en los docentes?}

El trabajo realizado por Mizala y Romaguera (2005) tuvo como metodología la realización de encuestas a profesores del Gran Santiago, en donde, frente a la aceptación de la evaluación y existencia de premios por desempeños, las respuestas son:

Un 74,7\% de los profesores está de acuerdo o muy de acuerdo con que el Ministerio de Educación debe aplicar un mecanismo de evaluación del desempeño de los establecimientos particulares subvencionados y municipales. Un $87,6 \%$ de los profesores está de acuerdo o muy de acuerdo en que es importante que se reconozca que hay establecimientos educacionales que se desempeñan mejor que otros. Hay un menor grado de acuerdo (aunque más de la mitad) con que el premio tenga efecto sobre la calidad de educación y la relación entre remuneraciones y evaluación. Un 55,6\% está de acuerdo o muy de acuerdo en que la entrega de un premio de excelencia por desempeño a los profesores contribuye a mejorar la calidad de la educación. (p. 548).

Respecto del análisis de la implementación y posterior desarrollo del SNED en la labor docente, se puede destacar ciertos cambios en la cultura tradicional docente, visualizada en una creciente aceptación por parte de los profesores de pruebas estandarizadas nacionales, más aún si éstas están ligadas con sus remuneraciones.

\section{4. ¿Son los incentivos (o aumentos) salariales símbolos de calidad educativa?}

Sin duda que uno de los temas recurrentes hoy en día, en políticas educativas, se relacionan con cómo fortalecer la calidad de la educación. La respuesta se puede dar usando diferentes enfoques; sin embargo, acá lo abordaremos, de forma breve, desde la perspectiva de los docentes y sus remuneraciones.

Hay que tener en consideración que el problema de los "bajos salarios" de los profesores es un problema demandado en toda Latinoamérica por parte del gremio (Mizala \& Nopo, 2012; Tenti \& Steinberg, 2007); no obstante, esta problemática social-laboral docente $i$ afecta $o$ influye en los procesos de calidad educativa? 
Sí, desde la perspectiva en que los bajos salarios y poca dispersión entre ellos impiden recular y retener a los mejores alumnos en las carreras de pedagogía; por lo tanto, es necesaria una política de carrera docente y de formación, que garantice condiciones laborales propicias y de remuneraciones. De esta manera, los bajos salarios tienden a desalentar el ingreso de buenos estudiantes a las carreras de pedagogía, afectando negativamente la motivación por enseñar, generando, como señala Díaz (2002), procesos de frustración en la tarea docente. También en algunos casos se genera el abandono por parte de los buenos docentes (Ayala, Ortúzar, Flores \& Milesi, 2015; Ruffinelli, 2014). Por lo tanto, un aumento salarial contrarrestaría los efectos anteriores, mejorando la calidad de la enseñanza. De acuerdo con Valenzuela, Muñoz, Silva-Peña, Gómez \& Precht (2015), se podría desarrollar una fuerte motivación escolar, resaltando así buenos resultados académicos. Para Mizala y Romaguera (2000b), "la evidencia empírica muestra que hay una fuerte vinculación entre la calidad de los profesores y el logro educativo de los alumnos, sin embargo, la evidencia acerca de la relación entre la calidad de los profesores y sus salarios no es concluyente" (p. 66).

Si analizamos desde la vereda contraria, es decir, que los bajos salarios no afectan los procesos de calidad de la enseñanza, surge como un argumento base que, si bien los incentivos y el aumento salarial ayudarían en interés de la pedagogía, eso no necesariamente discriminaría entre buenos y malos y profesores.

Si nos detenemos en observar las remuneraciones de los profesores en comparación con otros grupos de trabajadores (Mizala \& Romaguera, 2000a; Mizala \& Nopo, 2012) nos damos cuenta de que:

Al comparar los ingresos de los distintos grupos de trabajadores en forma bruta, es decir, sin considerar las diferencias de capital humano que éstos tienen, se encuentra que los profesores ganan en promedio más que el conjunto de trabajadores. Al comparar a los profesores con los grupos más educados de trabajadores, se observa que éstos ganan menos que los trabajadores que tienen 13 y más, y 17 y más años de educación. La brecha es, sin duda, muy grande con los individuos que tienen 17 y más años de educación, con quienes probablemente se comparan los profesores, lo que explicaría la percepción que ellos tienen de estar mal remunerados (Mizala \& Romaguera, 2000a, p. 26) 
Un problema que sí tienen las remuneraciones de los profesores en Chile es su falta de conexión con el desempeño de los maestros. Los salarios docentes son extremadamente parejos, independientemente del esfuerzo y el desempeño del profesor (Cox, 2005).

Claramente, si queremos tener una educación de calidad, se debe regular estas problemáticas a partir de políticas públicas que reconozcan la labor docente como relevante, y que forma parte de un de los procesos formativas más importantes: el de enseñar.

\section{Reflexión final, hacia una política de formación docente de calidad}

En esta sección del trabajo, y a modo de conclusión, conoceremos algunas propuestas de cuatro investigadores que ofrecen algunos lineamientos de una nueva política de formación docente, rescatando los elementos de estructura salarial y crítica de su quehacer como transmisores del conocimiento. Estos investigadores son Harald Beyer, Juan Eduardo García-Huidobro, Cristian Bellei y Beatrice Ávalos (ver tabla 1).

Tabla 1.

Investigadores e ideas en relación con la estructura salarial y la profesionalización docente en Chile.

Investigador(a) Principales ideas en relación a la estructura salarial

Harald Beyer A. La formación continua debe responder a una necesidad eviden(2007) te de un establecimiento para satisfacer los requerimientos que le impone un sistema de aseguramiento de la calidad exigente. La posibilidad de formación continua debe estar siempre presente, pero ella permitirá mayores remuneraciones siempre que signifiquen un impacto positivo sobre competencias pedagógicas.

B. Desde luego, seguir proponiendo un compromiso para asegurar a los profesores salarios que atraigan a las generaciones más jóvenes a la profesión docente -que, en proporciones demasiado significativas, privilegian otras disciplinas- e inviten a los buenos docentes actuales a mantenerse en la profesión.

C. Las definiciones salariales deben reconocer la escasez relativa en algunas disciplinas de la enseñanza por sobre otras y deben ir acompañadas de un reconocimiento de que es necesaria una mayor dispersión en los salarios entre profesores para asegurar la retención de profesores efectivos. En el mismo sentido, los umbrales salariales máximos deben subir, respecto de la situación actual, para aumentar los incentivos docentes.

D. Los aumentos de remuneraciones deben compatibilizarse adecuadamente con el sistema de financiamiento escolar.

E. El análisis de los profesores debe incluir, además, una reflexión más amplia sobre las condiciones de trabajo de los profesores (Beyer, 2007). 


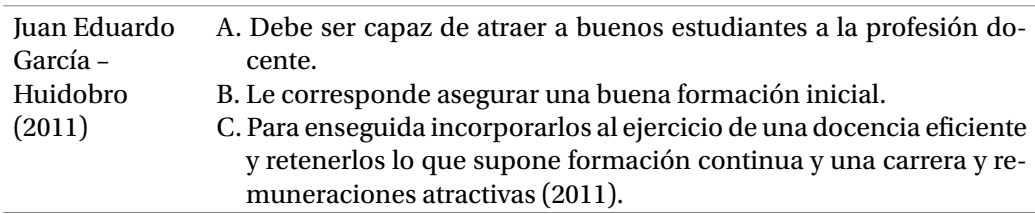

Cristian Bellei A. La estructura salarial debe progresar conforme a una combinación (2010) de experiencia y mérito; y debe permitir incentivar especialmente a docentes que se desempeñan en zonas aisladas o socialmente deprivadas.

B. El mérito debe ser ampliado y en ningún caso debe asimilarse mecánicamente a los resultados de la evaluación docente. En efecto, otro factor de diferenciación salarial es el desempeño de diferentes funciones en el establecimiento escolar.

C. Que las regulaciones y obligaciones salariales de la carrera rijan por igual en todo el sector subvencionado por el Estado, de lo contrario el impacto sobre el mercado laboral docente será limitado (2010).

Beatrice Ávalos A. Se establecen mejores condiciones de trabajo para los docentes, (2014) especialmente para aquellos que comienzan a enseñar, lo que se relaciona con la necesidad de establecer una formación inicial de calidad.

B. En lo que se refiere a políticas para estimular la retención de nuevos docentes, no hay hasta el momento una propuesta viable.

C. Hay buenas señales al momento de valorización de la profesión docente, expresadas en un creciente clamor porque se mejoren las condiciones de trabajo de los docentes y se establezca una carrera profesional digna para ellos. Significa también que declarar la obligatoriedad de un examen de conocimientos disciplinarios al egreso de la formación docente, sin verificar la capacidad práctica para enseñar, no asegura la calidad futura del ejercicio docente.

Fuente: elaboración propia, 2020.

Tal como se observa en la tabla 1, estos investigadores de diversas tendencias políticas y reconocidos en la investigación de política educativa, evidencian la importancia de establecer normativas y regulaciones que permitan crear una estructura salarial y de incentivo, con el fin de atraer profesionales y nuevos docentes, impactando en la calidad de la educación a partir del bienestar, motivación e innovación pedagógica. De esta manera, Beyer (2007) reflexiona que para alcanzar una educación de más calidad es necesario establecer nuevas instituciones, mirando hacia el futuro y no hacia el pasado. En ese marco, afirma la necesidad de asegurar incentivos y remuneraciones en base al aseguramiento de la calidad. Desde una mirada más crítica, García Huidobro (2011) afirma que "las remuneraciones son significativamente más bajas que en profesiones semejantes, lo que conlleva a una falta de reconocimiento social de la profesión"; a su vez, "las 
condiciones de ejercicio profesional son duras, la carga horaria es excesiva y no deja tiempo suficiente para planificar clases" (p.13). Por su parte, Bellei (2010), evidenciando los diversos documentos internacionales y nacionales, sostiene la importancia de una política integral de desarrollo docente. Finalmente, Beatrice Ávalos (2014) establece que todos los esfuerzos realizados por atraer buenos candidatos a la profesión docente y formarlos en la mejor forma posible, no traerán frutos si quienes egresan no se emplean en el sistema educativo, de ahí la importancia de establecer una formación inicial fuerte como también una política de remuneraciones que apunte con los estándares internacionales.

\section{Referencias}

Ávalos, B. (2002). Profesores para Chile. Historia de un proyecto. Santiago de Chile: Ministerio de Educación.

Ávalos, B. (2005). La formación de profesores y su desarrollo profesional. Prácticas innovadoras en busca de políticas. El caso de Chile, en C. Cox (Edit.), Políticas educacionales en el cambio de siglo. La reforma del sistema escolar. Santiago de Chile: Universitaria.

Ávalos, B. (2014). La formación inicial docente en Chile: Tensiones entre políticas de apoyo y control. Estudios Pedagógicos, 40(1).

Ayala, P., Ortúzar, M. Flores, C. \& Milesi, C. (2018). Búsqueda de empleo, proceso de inserción y dificultad de adaptación de profesores principiantes. Pensamiento Educativo. Revista de investigación educacional latinoamericana, 52(2).

Ball, S. y Youdell, D. (2008). Hidden privatisation in public education. London: Institute of Education.

Beech, J. (2006). Repensando la Transferencia Educativa: de la transferencia transnacional a los modelos universales de educación. Biblioteca Virtual Fundación Luminis

Bellei, C. (2015). Educación pública, educación privada y privatización, en El gran experimento. Mercado y privatización en la educación chilena. Santiago de Chile: LOM.

Bellei C. y Vanni, X. (2015). Evolución de las políticas educacionales en Chile: 1980-2014", en C. Bellei. El gran experimento. Mercado y privatización en la educación chilena. Santiago de Chile: LOM. 
Bellei, C. (2010). La carrera docente: mucho más que un esquema salarial, en Docencia, 42.

Beyer, H. (2007). Una educación de más calidad: algunas reflexiones. Revista Pensamiento educativo, 40(1).

Carnoy, M. (1999). Globalización y reestructuración de la educación. Revista de Educación, 318, 145-162.

Carnoy, M. y González, P. (2004). Reformas educativas y financiamiento en el Cono Sur (1980-2000), en: Las reformas educativas en la década de 1990: un estudio comparado de Argentina, Chile y Uruguay. Buenos Aires.

Carrasco, A. \& Flores, C. (2013). (Des) igualdad de oportunidades para elegir escuela: preferencias, libertad de elección y segregación escolar, en Espacio público, Documento de referencia 2.

Corvalán, J. y García Huidobro, J. E. (s/f.). Educación y mercado: el caso chileno. Santiago: Capítulo de libro en prensa.

Cox, C. (2005). Las políticas educacionales de Chile en las últimas dos décadas del siglo XX, en C. Cox (edit.) Políticas educacionales en el cambio de siglo. La reforma del sistema escolar. Santiago de Chile: Universitaria.

Díaz, A. (s./f.). Los procesos de frustración en la tarea docente. Docencia, 17.

Gajardo, M. (2012). La educación tras dos décadas de cambio. ¿Qué hemos aprendido? ¿Qué debemos transformar? en PREAL, 65, 1-20.

García-Huidobro, J. y Bellei, C. (2003) Desigualdad educativa en Chile. Santiago de Chile: Universidad Alberto Hurtado.

García-Huidobro, J. (2011). La política docente hoy y la formación de profesores. Docencia, 34.

González, P. (1999). Financiamiento de la educación en Chile. PREALUNESCO.

Hargreaves, A. y Shirley, D. (2012). Las tres vías del cambio, en A. Hargreaves y D. Shirley, La cuarta vía. El prometedor futuro del cambio educativo (pp. 25-50). Barcelona: Octaedro.

Mizala, A. \& Romaguera, P. (2000a). Remuneraciones y los profesores en Chile. Documentos de trabajo, 93, Centro de Economía Aplicada, Universidad de Chile.

Mizala, A. \& Romaguera, P. (2000b). Remuneraciones al pizarrón. $R e$ vista Perspectivas (Departamento de Ingeniería Industrial, Universidad de Chile), 4(1). 
Mizala, A. \& Romaguera, P. (2000c). Sistemas de incentivos en educación y la experiencia del SNED en Chile. Documentos de trabajo, 82, Centro de Economía Aplicada, Universidad de Chile.

Mizala, A. \& Romaguera, P. (2002). Regulación, incentivos y remuneración de los profesores en Chile. Documentos de trabajo, 116, Centro de Economía Aplicada, Universidad de Chile.

Mizala, A. \& Romaguera, P. (2003). Desafíos metodológicos de los sistemas de evaluación e incentivos en educación. El caso de SNED en Chile. Documentos de trabajo, 159, Centro de Economía Aplicada, Universidad de Chile.

Mizala, A. \& Nupo, H. (2012). Salarios de los maestros en América Latina: ¿Cuánto más o menos ganan sus pares?, en M. Cabrol y M. Székely (Edits.) Educación para la transformación, BID.

Mizala, A. \& Romaguera, P. (2005). Regulación, incentivos y remuneraciones de los profesores de Chile, en C. Cox (Edit.), Políticas educacionales en el cambio de siglo. La reforma del sistema escolar. Santiago de Chile: Universitaria.

Núñez I. (2002). Notas Históricas", en B. Ávalos Profesores para Chile. Historia de un proyecto. Santiago de Chile: Ministerio de Educación.

Programa Interdisciplinario de Investigación en Educación PIIE (1984). Las transformaciones educacionales bajo el régimen militar. Santiago de Chile: PIIE.

Ruffinelli, A. (2014). Dificultades de la iniciación docente: ¿iguales para todos?, en Estudios Pedagógicos, 40(1)

Tenti, E. (2011). Escolarización con pobreza: desarrollo reciente de la educación básica en América Latina, en La escuela y la cuestión social. Ensayos de sociología de la educación. Buenos Aires: Siglo XXI Editores.

Tenti, E. \& Steinberg, C. (2007). Características sociodemográficas y posición en la estructura social de los docentes en Argentina, Brasil y México. Pensamiento educativo, 42(2).

Valenzuela, J., Muñoz, C., Silva-Peña, I., Gómez, V. \& Precht, A. (2011). Motivación escolar: Claves para la formación motivacional de futuros docentes. Estudios Pedagógicos, 41(1). 